ing in this field and every medical scientist will find it full of interest. Professor Hardy, the Editor, is the Professor of Physiology at Yale and six other distinguished scientists including Professor Brobeck (Professor of Physiology at the University of Pennsylvania) and Professor Halzberg (Professor of Pathology at the University of Minnesota) have contributed chapters. Every aspect receives consideration; food and gaseous requirements, the problems of temperature, acceleration, weightlessness, isolation and perception and the sum total is a notable addition to scientific literature. In his foreword Professor Hardy points out that space engineers have from the outset endeavoured to provide for the astronaut the same environment that he has on earth but he stresses the importance of the role of physiologists and psychologists in defining the limits of human performance and survival in the stress of long periods of loneliness, extremes of heat and cold, high energy radiations, acceleration and high velocity and the vacuum of inter-planetary space. The mass of references reflect the enormous literature on space travel that has already accumulated. It is difficult to select any part of the book for especial mention as the standard of it all is so high, but the chapters on gaseous requirements, on temperature and on isolation and disorientation were of particular interest to the reviewer. In the last of these Dr. Randall Chambers from the U.S. Naval Air Development Center points out the importance of careful selection and training of astronauts, and of keeping them occupied during their flight.

\section{Problems of Biochemistry of the Nervous System}

Edited by A. V. Palladin. Translated from the Russian. Pp. xii +330 , illustrated. Oxford, London, New York and Paris: Pergamon Press. 1964. 80s.

This work is the English translation of the Russian book giving the contributions to a symposium held at Kiev in 1957 under the chairmanship of Professor A. V. Palladin. It is therefore highly technical in nature and can appeal only to the specialist. Such books generally have only a limited useful life, but since this book sheds light on the whole Russian approach to neurobiochemistry its interest may be greater than the seven year delay in publication might imply.

It is clear that many of the authors are dominated by the principles laid down by Pavlov, and that this affects the practice of medicine in Russia. We are told that sleep therapy is extensively used in Soviet clinics, although, rather tantalisingly, the actual uses are not stated. The objectives of the workers in this field are defined by Professor Palladin. "The task of functional biochemistry of the brain is to reveal correlations between specific functions of the nervous system and the chemical structure and metabolism of the nervous tissue . . " Some might quarrel with this aim, but we cannot ignore the results of the investigations which are presented. The functions studied however are mainly limited to metabolism of very simple substances such as glucose, ammonia, pyruvic acid, ATP etc., and under the influence of stimulation and depression of the brain by drugs, low temperature, and Pavlovian conditioned reflexes. Consequently, although much interesting factual matter is to be found, and generally the techniques used are advanced, many might think these methods too crude to advance the stated aims. Nevertheless the authors' attempt to correlate their results with function. Thus, we arrive at statements such as (p. 151) "The ammonia which forms when the drug is injected excites the central nervous system. Due to the continued action of ammonia this excitation becomes an inhibition which is accompanied by an increased amount of ammonia, due to its fixation into glutamine so characteristic of the inhibitory state". It is difficult to see just what is fact and what is deduction or assumption.

The translator and editors are to be congratulated upon having given us an English text which is both clear and agreeable in style.

\section{NEW EDITIONS}

\section{Practical Obstetric Problems}

IAN DONALD, M.B.E., M.D. (Lond.), F.R.C.O.G. Third Edition. Pp. xix +811 , illustrated. London: Lloyd-Luke (Medical Books). 1964. 70s.

The author has an easy and interesting style, e.g. he manages in this third edition to make a comparison of the two ways of performing transvaginal pudendal block read more like a description from an exciting novel, than like a dreary text. There are many good new things in this book. The ventouse is well described. The chapter on ante-partum hæmorrhage is excellent, although the speed of progress in obstetric thought has now overtaken Professor Donald's list of causes, because folic acid is not discussed fully as such an important cause as we now believe it to be. Psychoprophylaxis receives adequate mention.

\section{Gynæcology for Senior Students of Nursing}

John Cairney and J. CaIrney. Third edition. Pp. 247, illustrated. Christchurch, New Zealand: N. M. Peryer. 1963. 32s. 6d.

This edition includes for the first time chapters on hormone therapy and sex chromosome abnormalities, both of which are well written, whilst the chapter on contraception has been expanded to include an account of oral contraception. The technique of obtaining cervical smears and cervical cone biopsies is also described. The illustrations are well drawn although Figure 5 should be corrected as it shows the internal os wrongly placed and the lateral fornices non-existent.

\section{An Introduction to Electrocardiography}

L. Schamroth. Pp. $\mathrm{x}+165$, illustrated. 2nd Edition. Oxford: Blackwell Scientific Publications. 1964. 20s.

Dr. Schamroth is perhaps at his best when explaining rather complicated concepts. The genesis of the displaced ST segment in myocardial ischæmia is well described, and after a short perusal of the appropriate diagrams one is left wondering why the mechanism of the electrocardiographic patterns of bundle branch block had hitherto somehow avoided comprehension. Variations in the anatomical heart position and their effects on the electrocardiograph are simply described in diagramatic form. This second edition has been enlarged, and such topics as the Wolff-Parkinson White Syndrome, A-V dissociation and parasystole are now included. Recent work on phasic aberrant ventricular conduction, to which Schamroth has contributed, is particularly well described. 